\title{
Characterizing the Motion of Jointed DNA Nanostructures Using a Coarse-Grained Model
}

\author{
Rahul Sharma, ${ }^{\dagger}$ John S. Schreck, ${ }^{\ddagger}$ Flavio Romano, ${ }^{\circledR}$ Ard A. Louis, ${ }^{\S}$ and \\ Jonathan P. K. Doye*,\| \\ Department of Chemistry, Indian Institute of Technology Roorkee, Roorkee, 247667, India, \\ Department of Chemical Engineering, Columbia University, New York, USA, Dipartimento \\ di Scienze Molecolari e Nanosistemi, Universitá Ca’ Foscari Venezia, I-30123 Venezia, \\ Italy, Rudolf Peierls Centre for Theoretical Physics, University of Oxford, 1 Keble Road, \\ Oxford OX1 3NP, United Kingdom, and Physical and Theoretical Chemistry Laboratory, \\ Department of Chemistry, University of Oxford, South Parks Road, Oxford OX1 3QZ, \\ United Kingdom \\ E-mail: jonathan.doye@chem.ox.ac.uk
}

\section{Keywords}

DNA nanotechnology, self-assembly, molecular simulation, DNA origami, coarse-grained modeling

\section{Abstract}

As detailed structural characterizations of large complex DNA nanostructures are hard to obtain experimentally, particularly if they have substantial flexibility, coarse-grained modeling can potentially provide an important complementary role. Such modeling can provide a detailed view of both the average structure and the structural fluctuations, as well as provid-

\footnotetext{
*To whom correspondence should be addressed

${ }^{\dagger}$ Department of Chemistry, Indian Institute of Technology Roorkee, Roorkee, 247667, India

${ }_{\ddagger}^{\ddagger}$ Department of Chemical Engineering, Columbia University, New York, USA

"Dipartimento di Scienze Molecolari e Nanosistemi, Universitá Ca' Foscari Venezia, I-30123 Venezia, Italy

${ }^{\S}$ Rudolf Peierls Centre for Theoretical Physics, University of Oxford, 1 Keble Road, Oxford OX1 3NP, United Kingdom

"Physical and Theoretical Chemistry Laboratory, Department of Chemistry, University of Oxford, South Parks Road, Oxford OX1 3QZ, United Kingdom
}

ing insight into how the nanostructure's design determines its structural properties. Here, we present a case study of jointed DNA nanostructures using the oxDNA model. In particular, we consider archetypal hinge and sliding joints, as well as more complex structures involving a number of such coupled joints. Our results highlight how the nature of the motion in these structures can sensitively depend on the precise details of the joints. Furthermore, the generally good agreement with experiments illustrates the power of this approach, and suggests the use of such modeling to pre-screen the properties of putative designs.

DNA nanotechnology, in which DNA is used as a self-assembling engineering material to create structures and devices on the nanoscale, has seen spectacular progress over the last 15 years. ${ }^{1}$ The most widely-used approach has probably been DNA origami, 2 in which a long "scaffold" strand is folded up into almost any arbitrary structure made of arrays of double helices by the addition of many short "staple" strands. The first origamis, introduced by Rothemund in 2006, 3 were twodimensional, but approaches to generate 3- 
dimensional ${ }^{4}$ and twisted and bent ${ }^{[5] 6}$ structures soon followed. Origami's rise was further aided by the development of user-friendly computer-aided design tools. ${ }^{7}$ This structural control, coupled with origami's inherent addressability, has led to a diverse range of potential applications for DNA origami including drug delivery, ${ }^{8 / 12}$ nanoplasmonics, ${ }^{13 / 14}$ nanoelectronics, $\frac{15}{1}$ inorganic nanoparticle synthesis, 16 measurement of intermolecular interactions, $\frac{17 \mid 18}{18}$ and as nanopores,,$\frac{19}{22}$ rigid handles for tweezing, 23 nanosensors, 24 biosensors, 25126 and supports for protein cryoEM. 27

Here, our focus is on flexible DNA origami nanostructures. Typically, the arrays of DNA helices in an origami that are bound together by the crossing of strands at 4-way junctions lead to origamis having very stiff structures, particularly so for 3D origami. For example, the persistence length of an origami 6-helix bundle has been measured to be of the order of 1.9 $\mu \mathrm{m} .{ }^{28}$ To engineer flexible structures, distinct pseudo-rigid blocks are usually loosely coupled by single-stranded sections. Tensegrity structures, where the separate rigid origami blocks are held in place by long single strands under tension to give a well-defined overall structure, provide an extreme example of such a coupling. ${ }^{29}$ Often, the introduced flexibility is to allow angular motion, ${ }^{30}$ perhaps only after the induced unbinding of critical staple strands, as in the case of containers that open due to strand displacement ${ }^{3132}$ or aptamer binding. Similarly, the fixing of a flexible origami into a single configuration by molecular binding has been used as the basis for potential measurement devices. 18124

The Castro group have systematically explored the development of archetypal joints for DNA origami, e.g. a "hinge" that allows 1D angular motion, and a "slider" that allows 1D linear motion, including the tuning of their flexibility and range of motion by varying the properties of the single-stranded linking strands. $33-36$ These basic motifs can further be used as building blocks to render more complex mechanical behavior, such as crank-sliders, Bennett linkages, 34 and bi-stable switches. ${ }^{37}$ It is some of these jointed nanostructures that will be the focus of the current paper, because they provide representative examples of a wide class of flexible nanostructures.

Such nanostructures can naturally form the basis for devices that apply or measure mechanical forces. For example, the tension in DNA bridging the ends of a hinge origami has been utilized to study the unwrapping of nucleosomes. $38 \mid 39$ Similarly, the change in the equilibrium structure of a hinge origami has also been used to measure the interactions between nucleosomes. ${ }^{17}$ There have also been proposals for force-applying devices based on sliders. ${ }^{40}$

However, one of the difficulties in designing flexible origami structures is that it is hard to predict the flexibility and range of motion a priori, it being sensitive to the length and sequence of the usually single-stranded coupling strands, the excluded-volume constraints from the pseudo-rigid origami blocks and electrostatic repulsions. A major difficulty is the single-stranded sections. Firstly, once beyond a certain length there is the likely formation of secondary structure, such as hairpins. In principle, this could be avoided by choosing a sequence that would not form any secondary structure, however, sequence is not usually an available variable for origami design, as it is fixed by the viral scaffold. Secondly, even without secondary structure, simple polymer models such as the freely-jointed chain or the worm-like chain cannot fully capture the behavior of ssDNA, because of the combined effects of excluded volume and the stacking interactions between bases. For example, as fullystacked single-stranded DNA (ssDNA) is effectively shorter than unstacked ssDNA, and the degree of stacking varies substantially as a function of the tension in the strand, 41 its contour length is not a well-defined property independent of the degree of stacking.

One of the most common ways to measure the flexibility of origamis experimentally is to characterize many, say TEM, images of the structures on a surface, thus allowing one to obtain probability distributions for the structural variable of interest (e.g. angle for a hinge origami). Aside from possible limitations due to resolution and the possible effects of the surface or 
staining on the origami geometry, there is a more fundamental issue of heterogeneity. As the measurements are on ensembles of structures, rather than following the motions of a single device, some of the variation in a property might be due to heterogeneity in the population of devices due to structural imperfections (e.g. missing or incorrectly-bound staples) rather than the intrinsic range of motion for the ideal defect-free origami. Although the quality of origami structures has been studied for some examples, ${ }^{42}$ how close experimentally-realized origamis are to their ideal designs is still very much an open question.

Small-angle x-ray scattering provides an interesting alternative structure determination method and has the potential to provide information about origami structures in solution. $\stackrel{43144}{ }$ However, the resulting curves usually have to be compared to the predictions from a structural model, and will also reflect any structural heterogeneity.

Therefore, modeling has potentially a particularly significant role in helping to understand flexible origamis both by visualizing their structures and range of motion, and by providing a greater microscopic understanding of how the behavior can be modified by changes to the design. All-atom simulations would be desirable, as they can potentially provide the greatest level of detail. However, although such simulations have been performed for a number of examples, 21/45/46 they are computationally extremely demanding and this cost means that they cannot be routinely performed. Furthermore, so far all-atom simulations have only been applied to relatively stiff origami. Simulating over long enough time scales to capture the motion of a flexible origami would be an extreme challenge.

Towards the opposite end of level of detail and computational cost, "CanDo" uses a finiteelement approach to model origami as elastic rods (representing the helices) connected by different motifs, such as 4-way junctions, whose mechanical properties have been parameterized. $47 \sqrt[50]{ }$ By minimizing the energy in the model, the mechanical equilibrium structure can be obtained, and by computing the nor- mal modes of this equilibrium structure the fluctuations of the structure can be estimated using equipartition. $\frac{48}{}$ More recently, a Brownian dynamics approach has been developed to provide dynamical trajectories for the model. CanDo can provide rapid and reliable predictions of origami structure, and so has proved to be an extremely useful and widely-used tool for the DNA nanotechnology community. However, CanDo cannot so usefully be applied to the flexible structures considered here, chiefly because it does not have any explicit representation of excluded-volume interactions, which play a key role in constraining the motion in these flexible structures (see Fig. S1).

In between these all-atom and mechanical models are coarse-grained models at the nucleotide level that start with a simplified representation of the internucleotide interactions. Although there are a wide number of such models, $, 51,57$ here we use the oxDNA model $41|58| 59$ as it has been parameterized with DNA nanotechnology in mind, and has been very successfully used for a wide variety of such applications. 60 In particular, it has been shown to be able to accurately reproduce the basic mechanical properties of duplex ${ }^{[5968] 69}$ and single-stranded ${ }^{41}$ DNA, as well as the structure of "stiff" origamis, ${ }^{59 / 67 / 70}$ making it ideally suited to study the jointed origami nanostructures that are the focus of this paper. Mention should also be made of a recently developed pseudo-rigid body model at an even more coarse-grained level of detail specifically developed for the jointed nanostructures of the Castro group that treats each section of the origami as an elastic object. $\underline{52}$

Specifically, we will consider the basic hinge and sliding joints developed by the Castro group, and their combination in a crank-slider where angular and linear motion are coupled, and in a Bennett linkage that has coupled angular motion. ${ }^{34}$ We will particularly focus on how the flexible coupling between the different sections plays a critical role in the observed behavior. Furthermore, the generally good agreement between our simulations and experiment shows the potential for oxDNA to be used to prescreen putative origami designs to check they have the 
required properties prior to experimental realization.

\section{Results and Discussion}

Our basic approach is simple, but computationally demanding. We run multiple molecular dynamics simulations of each design until the total simulation time is sufficient to provide wellconverged distributions for the slowly-varying structural descriptors that we use to characterize the flexible motion of the nanostructures. For example, in Fig. S3 we show the fluctuations of the hinge angles with time to illustrate the time scales associated with the relative diffusive motion of the two halves of these hinges. Movies for example trajectories for each design are also available as part of the Supporting Informaiton.

Rotary motion: Hinges. We have studied two versions of rotationally constrained hinges that were studied experimentally in Ref. 34 and which are illustrated in Fig. 1. They are composed of two 18-helix bundle (hb) stiff arms $(3 \times 6$ square lattice $)$ which are aligned along an edge by flexible ssDNA scaffold connections. There are two types of ssDNA connections both of which connect helices adjacent to the axis of rotation. Firstly, there are three short 2nucleotide (nt) scaffold connections between nucleotides that are at the edges of the bundles, and which effectively define the rotation axis. Secondly, there are three longer connections (16 nt and 30 nt for "hinge-1" and "hinge-2", respectively) between nucleotides on the opposite sides of the helices, which act to constrain the motion of the hinge. For the case of hinge-2 there are also two 12-nt staples that bind to each of the longer connectors to provide a physical constraint preventing secondary structure. The connectors are highlighted in the close-ups of the hinge region in Fig. 11(c-f). For both the hinges, there are also two rows of helices on the lower arm which provide a physical constraint disfavoring the opening of the hinges to very large angles.

Example configurations for both hinges are shown in Fig. 1(a) and 1(b) with the probabil- ity distributions for the hinge rotation angle $\theta$ in Fig. 2, The two hinges have dramatically different angle distributions with hinge-1 being more open and much more flexible, and hinge- 2 favoring more closed configurations and having a much narrower range of motion.

The effects that disfavor more closed configurations are electrostatic repulsions between the origami arms, and the loss of entropy resulting from a more extended configuration of the longer connector. The latter is more significant in hinge- 1 both because of the shorter connectors and their purely single-stranded character - the alignment of the two 12-bp double helical sections in each hinge- 2 connector requires less loss in entropy.

The factors that disfavor the more open state of the hinges are the loss of conformational entropy of the longer connectors, this time as they become confined to a smaller volume between the ends of the two arms, the self-interactions in the connectors that this confinement induces, and for very open configurations direct repulsive interactions between the two arms. The confinement-induced interactions are particularly problematic for hinge-2, as is evident from Fig. 1(b) and 1(f), as the small single-stranded gaps between the two duplex sections for each connector make it difficult for the duplexes to avoid each other, and are likely to be the main reason this hinge only adopts relatively small hinge angles.

If we now compare our results to the experimental distributions in Ref. 34, our results for hinge-1 look promising, with the position of the maximum at a very similar angle $\left(\sim 70^{\circ}\right.$ for oxDNA compared to $\sim 80^{\circ}$ in the experiment). The main difference is that the experimental distribution is slightly wider and more symmetric with very open configurations more probable. We note that the effect of the potential heterogeneity in the experimental origamis, mentioned in the introduction, would most likely to lead to greater variation in a property.

By contrast, our results for hinge-2 are very different from experiment. Both experiment and simulation find hinge-2 to be more closed than hinge-1, but oxDNA's most likely hinge angle is $30^{\circ}$ smaller than experiment and the 
(a) Hinge-1, $\theta=68.4^{\circ}$

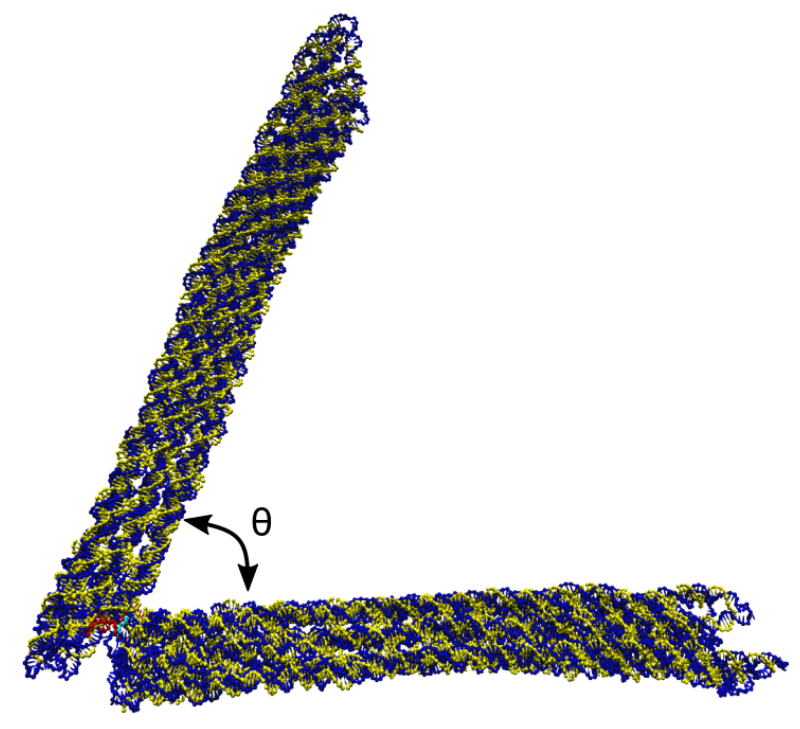

(b) Hinge-2, $\theta=27.9^{\circ}$

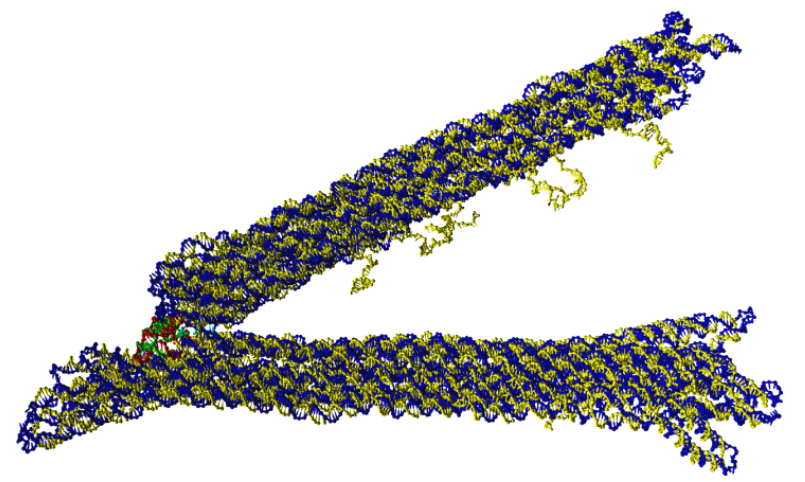

(c) Hinge- $1, \theta=13.0^{\circ}$

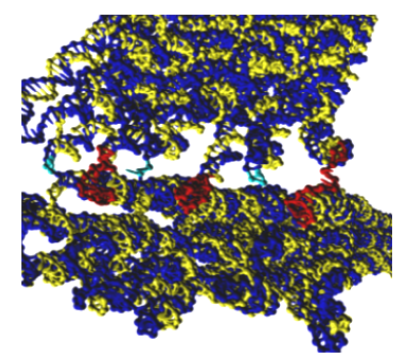

(e) Hinge-2, $\theta=13.1^{\circ}$

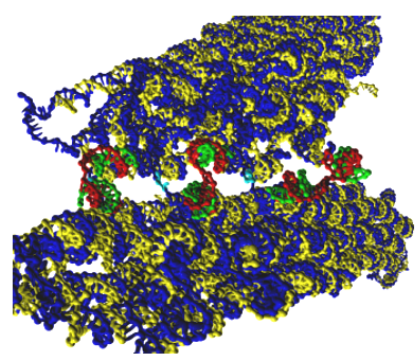

(g) Hinge-2, $\theta=16.7^{\circ}$ (without staples)

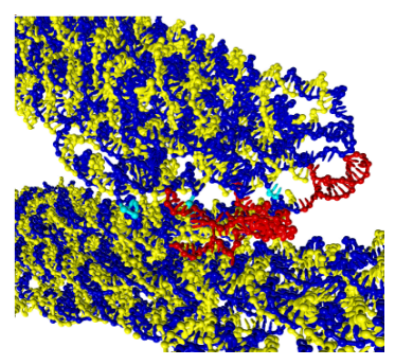

(d) Hinge-1, $\theta=103.4^{\circ}$

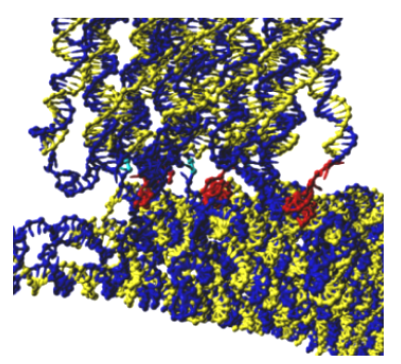

(f) Hinge-2, $\theta=53.4^{\circ}$

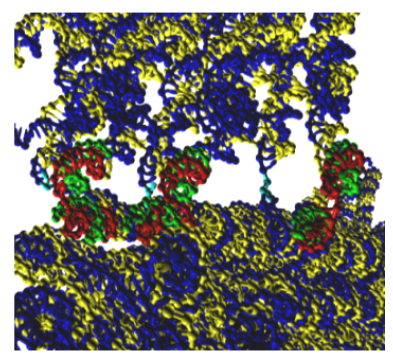

(g) Hinge-2, $\theta=84.7^{\circ}$ (without staples)

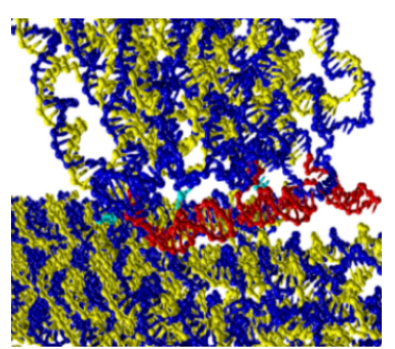

Figure 1: Typical configurations for (a) hinge-1 and (b) hinge-2. (c and d), (e and f), and (g and h) provide a close-up of the hinge region of hinge- 1 , hinge- 2 , and hinge- 2 without the single-domain staples that are designed to bind to the longer connecting strands, respectively. The scaffold strand is dark blue and staples yellow with the very short connecting strands at the hinge joint in cyan, longer scaffold connectors in red and any single-domain staples binding to the connectors in green. The full definition of the hinge rotation angle $\theta$ is provided in Fig. S2. 

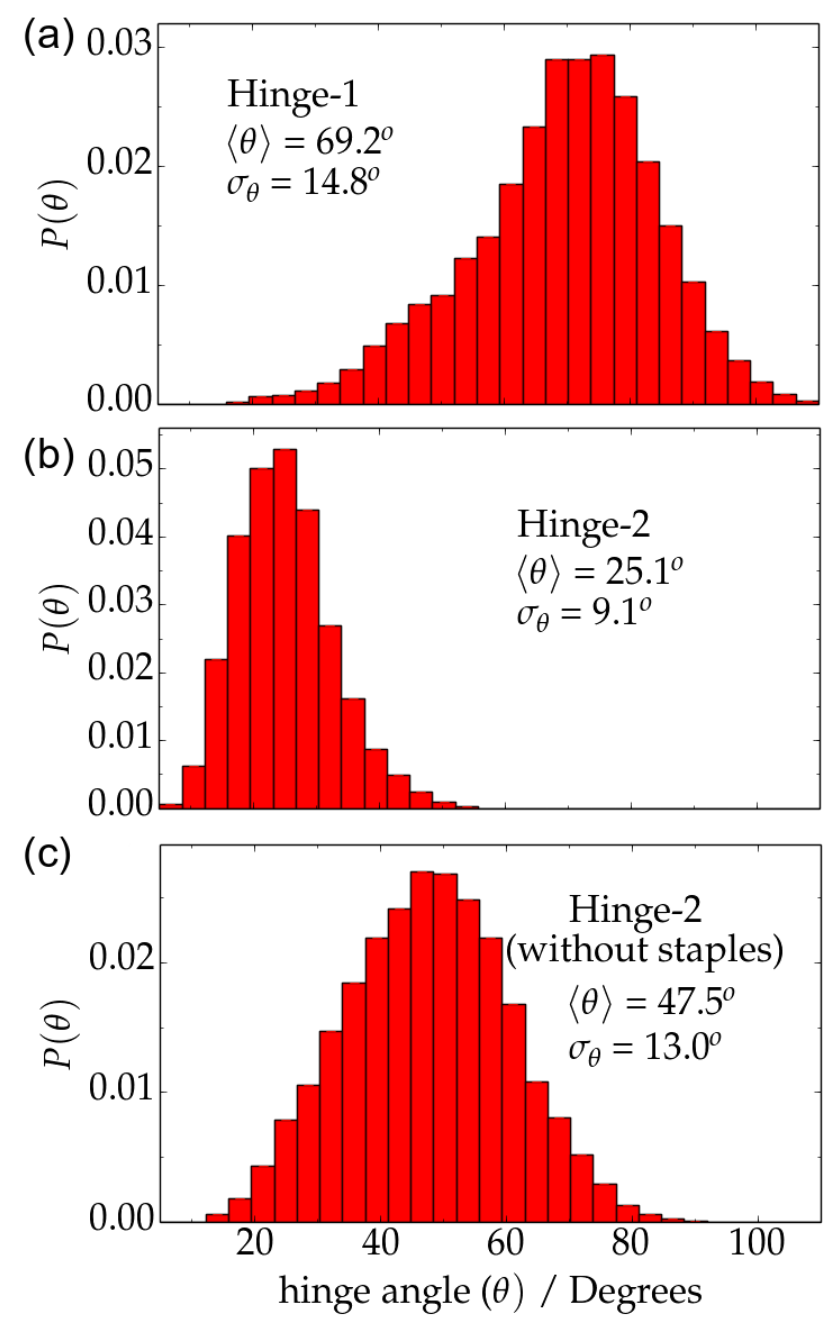

Figure 2: Probability distributions of the hinge angle for (a) hinge-1, (b) hinge-2 and (c) hinge2 without the single-domain staples bound to the single-stranded connectors between the two halves of the hinge. Also given are the mean and standard deviations of the distributions. distribution is very much narrower. What could be the cause of this big difference? Given oxDNA's numerous quantitative successes at reproducing the geometric and mechanical properties of DNA and its assemblies, $\frac{66 / 67 / 71}{1 t}$ seems unlikely to us that it is simply due to some major failing in the model. This is particularly so given the similarity of the simulation and experimental results for hinge- 1 .

In the experiments, prior to imaging, the origamis are purified by electrophoresis, thus separating them from the excess of staples. Thus, if a staple dissociates from the origami during or after this process it is unlikely to be replaced. This is particularly pertinent to the short 12-nt single domain staples that are designed to bind to the connecting strands. As the interactions involving these duplex sections are the major reason for more open states of the hinge being unfavored, their absence in the imaged origamis could be the reason for the differences between the oxDNA and experimental distributions.

To test this hypothesis, we also simulated hinge- 2 but without any staples bound to the longer connectors. The resulting angle distribution is presented in Fig. 2(c) along with example configurations in Fig. S4. The hinge now both adopts a much more open configuration and is much more flexible. Indeed, the most likely angle $\left(\sim 50^{\circ}\right.$ for oxDNA) is essentially the same as for the experimental distribution, although, similar to hinge-1, the simulation distribution is slightly narrower. Thus, these results provide significant support for our suggestion that the staples that are designed to bind to the connecting strands are not present in the imaged hinges.

Looking at the detailed configurations of the single-stranded connectors it is apparent that when in a more open state they have a strong tendency to form hairpins, whereas they form much less secondary structure when in a more closed configuration (Figs. 1(g) and 1(h)). Thus, this secondary structure is one of the drivers for the hinge to adopt a more open configuration.

Linear motion: Sliders. Next, we consider the two sliders investigated in Ref. 34 . 
(a) Slider-1, $\mathrm{d}=63.6 \mathrm{~nm}$

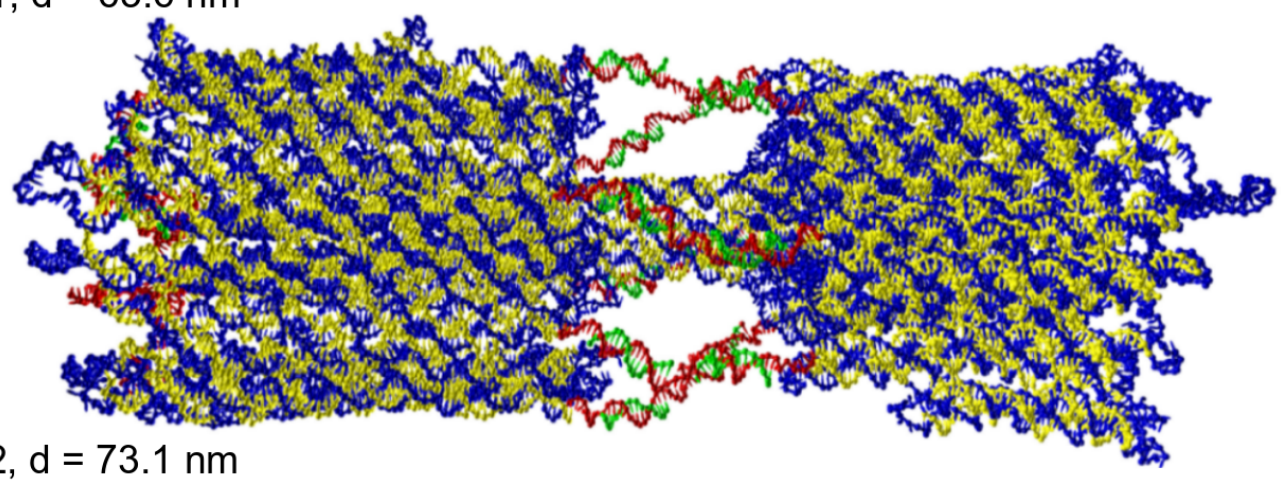

(b) Slider-2, $\mathrm{d}=73.1 \mathrm{~nm}$
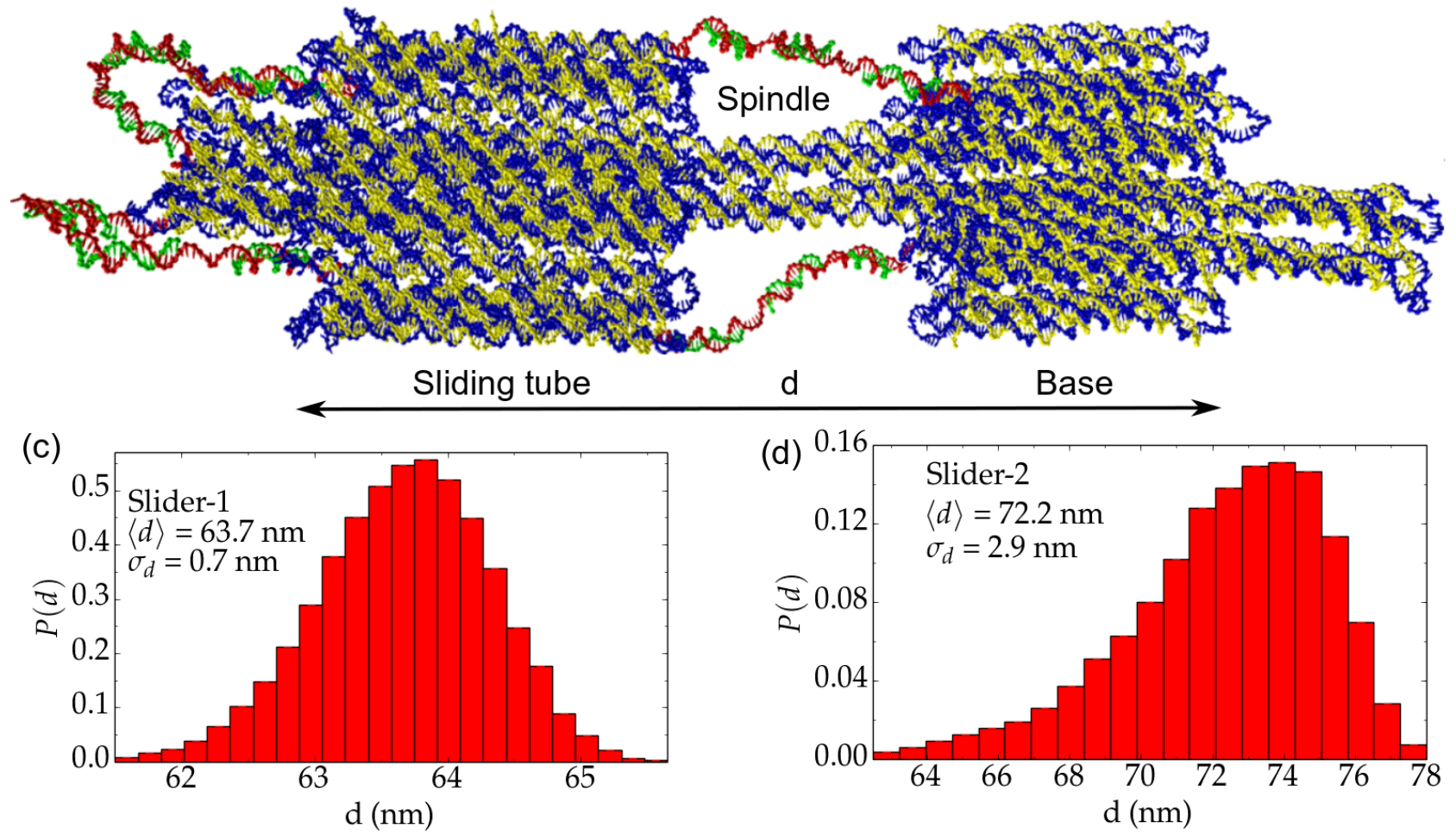

Figure 3: (a) and (c) represent an average configuration for slider-1 and slider-2, respectively, while (b) and (d) give probability distributions for the extension. The same color scheme is used as in Fig. 1. The extension is defined as the distance between the far ends of the sliding tube and the base (see also Fig. S6). 
The structures, which are illustrated in Fig. 3. consist of an inner 6-helix bundle that is surrounded by an outer sliding tube of 30 helices. The translational motion of the tube is restricted by a 42 helix-bundle at the base of the 6-helix-bundle spindle and also by singlestranded connectors between the sliding tube and both the base and the end of the spindle. In the two versions of slider, the number, length and nature of these connectors differ, as well as the length of the spindle, facilitating different ranges of linear motion. In "slider-1", the sliding tube is connected to the base by six 49nucleotide connections and to the other end of the spindle by six 28-nt connections. By contrast, "slider-2" has two 86-nt connections between the sliding tube and the base and two 96-nt connections between the tube and the end of the spindle. As with hinge-2, single-domain staples that bind to these connecting strands are also part of the design. However, they will also significantly affect the mechanical properties of these linkers. Rather than exhibiting the force-extension behavior of single-stranded DNA, these connectors are better thought of as a series of rigid double helical units connected by short flexible single-stranded sections, and will have a significantly smaller effective force constant than single-stranded DNA. In version2 of the slider all of the connectors have three 18-20 nt staples bound to them, whereas in version-1 each of the connectors between the sliding tube and the base have two 15-nt staples but the connectors at the other end of the slider are single-stranded.

A typical configuration for slider- 1 is shown in Fig. 3(a). It is noticeable that the sliding tube is held close to the end of the spindle. This simply reflects the much greater stiffness of the single-stranded connectors between the spindle end and the tube compared to the staple-covered connectors between the tube and the base. Consequently, the extension distribution (Fig. 3(c)) is comparatively narrow reflecting the relatively small thermal fluctuations in the position of the sliding tube. In particular, the tube never gets close to the base; their average separation is $11.7 \mathrm{~nm}$ with a standard deviation of only $0.7 \mathrm{~nm}$ (Fig. S8(a)).
Comparing to the experimentally-measured extension distribution, although the maximum extensions are similar, in experiment the slider was found to exhibit a much wider range of extensions with some configurations with the tube very close to the base. Given the simple physical origin of the narrow extension distribution and the general ability of oxDNA to capture DNA's mechanical properties, to us this implies that the experimentally observed origamis cannot all have the ideal structure. In particular, a loss of any of the 15-nt single-domain staples on the connectors between the tube and the base would lead to an increase in their stiffness and hence a shift of the extension to shorter distances.

A typical configuration of slider-2 is shown in Fig. 3(b), and the extension distribution in Fig. $3(\mathrm{~d})$. Firstly, the extension is larger than for slider-1 simply due to the longer spindle in this case. Secondly, because of the fewer number of connectors and because all are staple-covered, the extension distribution is significantly wider than for slider-1. Interestingly, even though all the connectors are similar, the sliding tube is likely to be found closer to the end of the spindle (average separation $13.9 \mathrm{~nm}$ ) than to the base (average separation $19.6 \mathrm{~nm}$ ) (see also Fig. S8). The configuration in Fig. 3(b) gives us a clue to this difference. The connectors between the tube and the base must lie between these two objects, whereas the connectors to the spindle can project into the empty space beyond the end of the spindle.

Comparing to experiment, our results capture the greater range of motion in slider- 2 than in slider-1, however our extension distribution is still significantly narrower than experiment. Again we suggest that this is likely due to a heterogeneous population of origamis in the experiment.

Coupled motion: a) Crank-slider. The next origami that we consider, a so-called "crank-slider", couples linear and angular motion and consists of three hinge joints and one sliding joint; it is illustrated in Fig. 4(a). The hinge joints are similar to those of hinge- 2 discussed above and consist of two 2-nt ssDNA scaffold connections that define the hinge-axis 
(a) Crank-Slider

$\theta=66.4^{\circ}, x=36.4 \mathrm{~nm}$

(b)

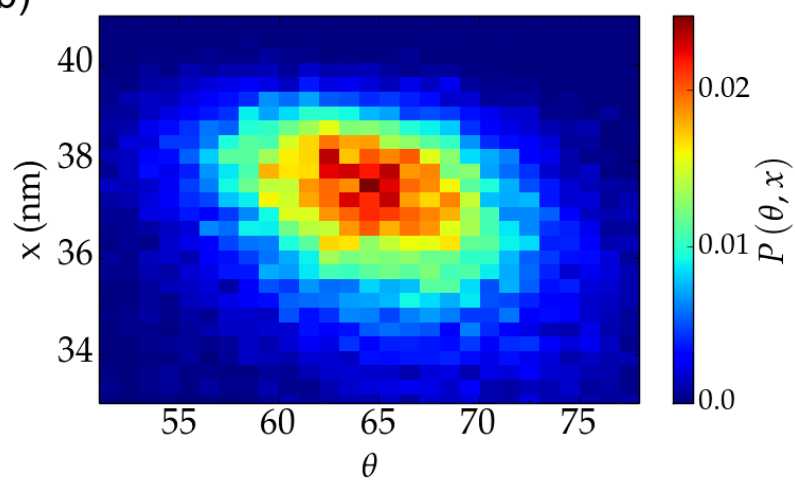

(c)

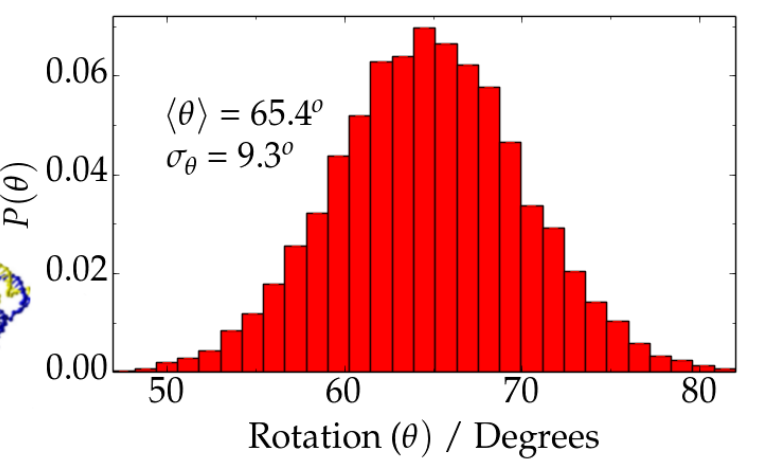

(d)

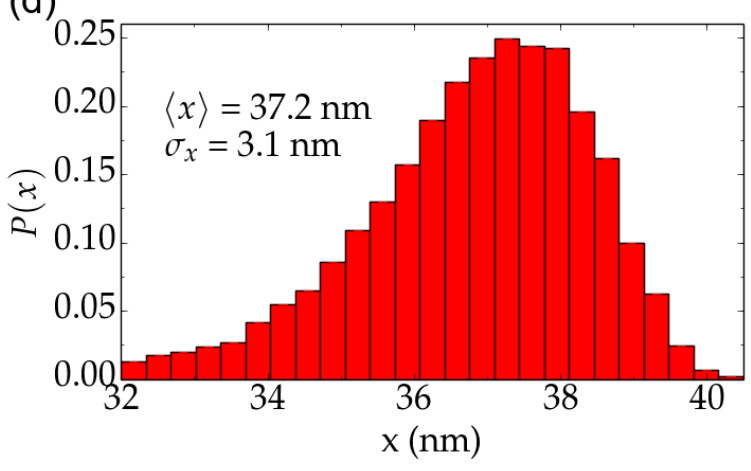

Figure 4: (a) A typical configuration of the crank slider. (b) Joint probability distribution for the hinge angle $\theta$ and extension $x$, plus individual distributions for (c) $\theta$ and (d) $x$. Fuller definitions of these quantities are given in Fig. S10. 
and two longer 34-nt connections between the neighboring helices. The sliding tube is connected through two 52-nt connections to the base and through two 77-nt connections to the end of the spindle. Also, to prevent hairpin formation in the longer hinge connections and the slider connections, complementary staples of 12 nt and 16-20 nt have been used, respectively.

The coupling between the different joints constrains their behavior. For example, the angles at the three hinge joints must add up to roughly $180^{\circ}$. Thus, their average angles must be significantly larger than that we found to be preferred for the hinge-2 joint above.

One might expect that the combined effect of the force constants of the individual joints will be additive and tend to make the coupled system significantly stiffer than that of the individual joints. However, that the variables (i.e. hinge angle or extension) associated with the multiple free-energy terms cannot all adopt their optimal values in the preferred geometry of the coupled system leads to a flattening of the landscape. For example, as the slider moves, some of the hinge angles will move towards their more preferred angle, while others will move away. These effects are seen in our results. The distribution for the chosen hinge angle (Fig. 4(c)) has a very similar width to that of hinge-2, while the extension distribution (Fig. 4(d)) is actually wider than slider-2 (its closest analogue).

From the 2-dimensional free-energy landscape (Fig. 4(b)), the coupling between the slider extension and the chosen hinge angle is clear, namely as the slider contracts the angle between the base and the connecting arm must increase. This coupling is also very apparent from the additional more extreme configurations illustrated in Fig. S11. The coupled motion, as indicated by the direction of softest curvature in Fig. 4(b), is also very similar to that observed experimentally and that expected on simple geometric grounds. $\frac{72}{}$

When comparing the distributions to experiment less can be said than for the hinges and sliders because of the fewer number of experimental data points. However, our data is fairly similar with again a somewhat larger spread of extension and hinge angle in the experiments. Like with slider-2 we think that it is unlikely that the 12-nt staples that are designed to bind to the hinge connectors would be present under observation conditions.

Finally, if we look at the detailed configurations in Fig. 4(a) and Fig. S11, one can sometimes see that the bending is not fully localized to the putative hinge axis. Instead, there is a tendency for the helices connected by the short connectors to bend away from their arms at the nearest 4-way junction to the hinge.

b) Bennett Linkage. The last case study that we consider is the Bennett linkage origami created in Ref. 34 and illustrated in Fig. 5. It consists of four arms and four hinges, with the hinges on different planes thus allowing all four hinges to close simultaneously in a cooperative fashion to generate a compact closed configuration. The hinges, unlike those considered so far, just consist of 2-nt connections on the hinge axis with no further constraining singlestranded connectors. As such the hinges would be expected to be individually relatively free in the angles they adopt. Each arm is a $4 \times 4$ 16-helix bundle.

For the other origamis considered so far the motion of the nanostructures occurs roughly in a plane. However, the 3-dimensional motion of the Bennett linkage makes it much harder for this motion to be fully characterized from the experimental microscopy images; a kinematic analysis approach $\frac{73 / 74}{15}$ ised to infer as much information as possible. ${ }^{72}$

We find that on average the linkage wants to be roughly planar, although there are substantial fluctuations from planarity in both directions (Fig. 5(c)). However, these fluctuations are still a long way from a fully folded configuration. This differs from the experiments where, although the majority of configurations seem to adopt a relatively open state, there are some which exhibit substantial folding. 72

The probability distribution of Fig. 5(d) shows the coupled nature of the motion of the Bennett linkage for the two angles that were directly measured in experiment, with a very similar behavior being found. We can also examine the coupling between the internal angles of the 


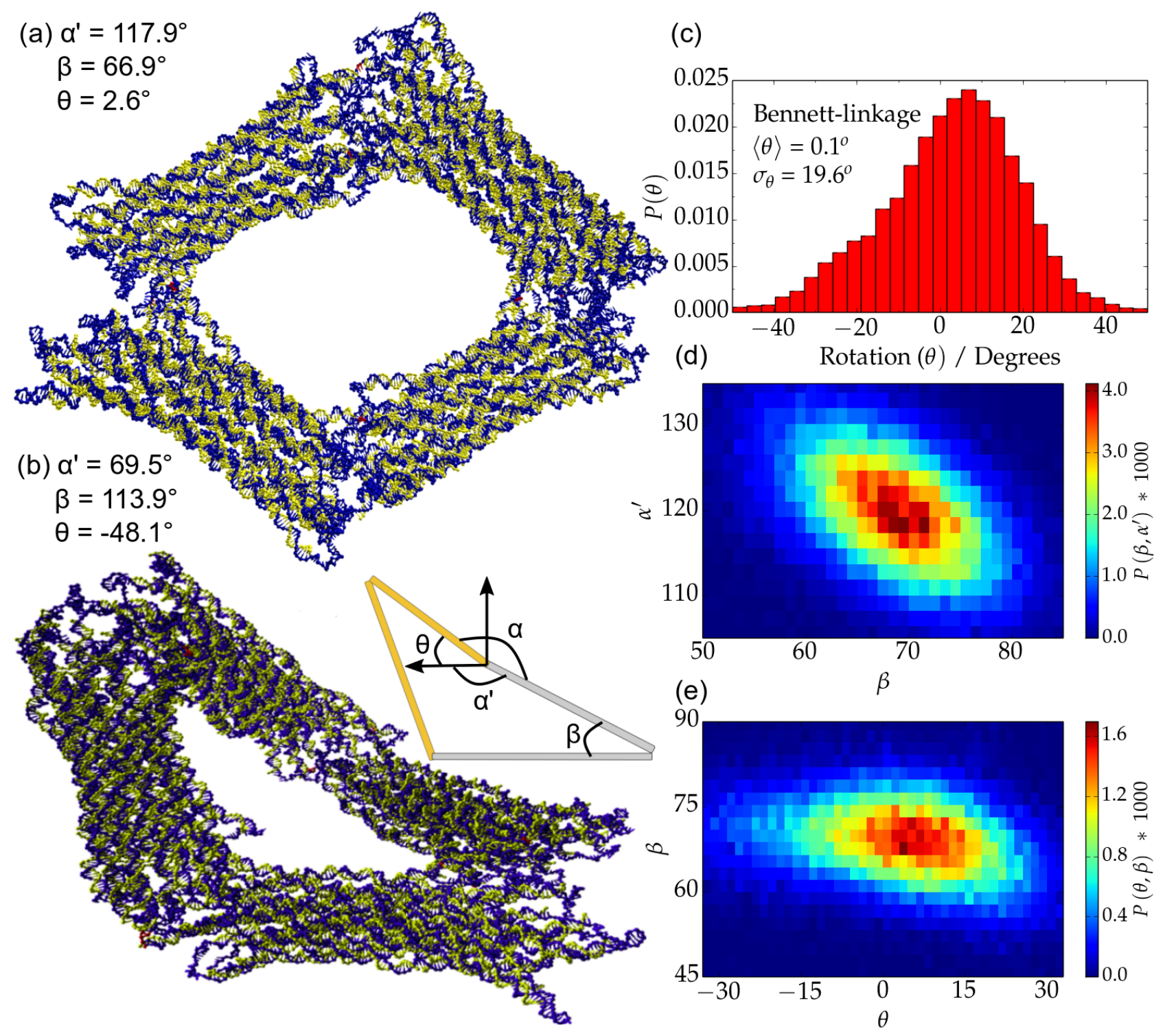

Figure 5: (a) A typical relatively flat configuration of the Bennett linkage. The hinges axes are roughly perpendicular to the plane for the hinges on the left and the right, and in the plane for the hinges at the top and bottom. (b) An example of a non-planar configuration. (c) Probability distribution for $\theta$, where $\theta$ is a measure of the non-planarity of the structures. (d) The joint probability distribution of the angles $\beta$ and $\alpha^{\prime}$, which are measures of the internal angles of the polygonal shape. (e) Joint probability distribution for angles $\beta$ and $\theta$. The exact definitions of all measured angles are given in Fig. S12. 
linkage and the non-planarity of the structure. For example, Fig. 5(e) shows how the smaller internal angle decreases as the linkage fluctuates out of the plane to positive values of $\theta$, consistent with it adopting a more closed configuration (a fully closed configuration would be expected to have $\theta=180^{\circ}$ and $\beta=0^{\circ}$ ).

\section{Conclusions}

Here, we have again demonstrated the ability of the oxDNA coarse-grained model to accurately capture the structural properties of large DNA nanostructures, $\frac{596166616770}{1 n}$ in this instance for jointed DNA nanostructures where the flexibility is a particular challenge. Aspects of this challenge include the long time scales associated with the relative diffusive motion of the quasi-rigid components and the sensitivity of the range of motion to the precise details of the design of the joints. For the latter, it is important that the model is able to well describe the properties of single-stranded DNA, such as its force-extension behavior, its ability to form secondary structure and the excluded-volume it possesses. The ability to naturally capture the unstacking that can occur at nicks and 4-way junctions, and which allows helical sections to bend considerably if relatively unconstrained by other junctions as is sometimes the case near to the joints of these nanostructures, may also be important. These are all features that simpler alternative tools may struggle to capture.

Our simulations of these jointed nanostructures are able to provide a much more detailed visualization and characterization of their structure than is possible through experimental microscopy, including well-converged probability distributions for the key degrees of freedom associated with their motion, and joint distributions that show the nature of the coupled motion in the crank-slider and Bennett linkage. This is particularly so for the Bennett linkage where the 3-dimensional nature of the coupled motion of the linkage means that it is harder to infer from the experimental images.

These kinds of simulations can also provide physical insight into the role of design features on the structural properties. This is particularly the case for the connectors at the joints, where we have shown how small changes to the design, such as the presence or absence of short single-domain staples bound to the connectors, can dramatically alter the motion. For example, these single-domain staples not only inhibit secondary structure formation but also significantly change the mechanical and excludedvolume properties of the connectors.

The comparison of our results to experiment is generally good. The main differences are that the range of motion that we observe in our simulations is generally somewhat more limited than in the ensemble of structures in the experimental microscopy images. Although we cannot rule out that this could simply be due to deficiencies in the oxDNA model, given the previous successes of oxDNA in capturing the structural properties of large DNA nanostructures and that the motion of these structures depends on DNA properties that oxDNA captures well, we feel that the main source of these differences lies elsewhere. In particular, we should only expect the time-averaged properties of our single ideal origami to be equivalent to that of the experimental ensemble when all of the origamis are assembled completely correctly. Instead any heterogeneity in the ensemble of origamis will lead to a broadening of the distributions since they will represent a sum over a set of distributions for the different structures present. Potential sources of assembly defects that would not necessarily be evident from the electron microscopy images include missing staples (as we have shown for hinge-2, such defects can potentially have a very significant effect on the properties when associated with the joints) or more subtle defects associated with an incorrect routing of the strands that gets locked in during the assembly. This study also confirms the potential utility of using oxDNA simulations in not only rationalizing some of the behaviour of already realized structures, as here, but also in aiding the design process of complex DNA nanostructures by testing the properties of putative designs. 


\section{Methods}

In this work, we use oxDNA, a coarse-grained DNA model at the nucleotide level. ${ }^{4158159} \mathrm{In}$ particular, we use the latest version of the model ("oxDNA2") that has had the pitch and the properties of nicks and junctions finetuned in order to better reproduce the structural properties of DNA origamis. ${ }^{59}$ In oxDNA, each nucleotide is represented by a rigid body and the nucleotides interact with an effective potential that has terms representing backbone connectivity, excluded-volume interactions, hydrogen bonding between base pairs, stacking interactions and electrostatic interactions between backbone phosphate groups. Solvent is modeled implicitly as a dielectric continuum, and we choose to use a salt concentration of $\left[\mathrm{Na}^{+}\right]=0.5 \mathrm{M}$, which is representative of the high salt conditions typically used for DNA nanotechnology.

To generate configurations for the origami structures, the original cadnano files were converted to oxDNA format. The generated configurations are typically not yet suitable as starting points for molecular dynamics simulations, as, due to their representation within the cadnano file, some nucleotides may be subject to extremely large forces due to particle overlaps or extended bonds. Therefore, the configurations are first "relaxed" using a specially-adapted minimization algorithm. In order to simulate large origamis with thousands of nucleotides within a reasonable computational time, we use a GPU-enabled version of our simulation code. ${ }^{755}$ To reproduce the effect of the solvent in generating Brownian motion of the nucleotides, we use a Langevin-like dynamics algorithm based on a strongly-coupled Andersen thermostat. ${ }^{767}$ Due to the flexibility and range of motion allowed for the origamis studied here, particularly long runs were required to ensure converged probability distributions of structural properties. For example, the sliders were simulated for over 10000 GPU hours.

Conflict of Interest: The authors declare no competing financial interest.

Acknowledgements. We are grateful to the Engineering and Physical Sciences Re- search Council (EPSRC) for financial support. We acknowledge the use of the University of Oxford Advanced Research Computing (ARC) facility in carrying out this work, http://dx.doi.org/10.5281/zenodo.22558 . We are grateful to Carlos Castro for supplying us with the original cadnano files.

Supporting Information: The Supporting Information is available free of charge on the ACS Publications website at DOI: 10.1021/acsnano .

The precise definitions of all measured angles and extensions and additional simulations results. Animations of trajectories for each of the jointed nanostructures.

\section{References}

1. Zhang, F.; Nangreave, J.; Liu, Y.; Yan, H. Structural DNA Nanotechnology: State of the Art and Future Perspective. J. Am. Chem. Soc. 2014, 136, 11198-11211.

2. Linko, V.; Dietz, H. The Enabled State of DNA Nanotechnology. Curr. Opin. Biotechnol. 2013, 24, 555-561.

3. Rothemund, P. W. K. Folding DNA to Create Nanoscale Shapes and Patterns. Nature 2006, 440, 297-302.

4. Douglas, S. M.; Dietz, H.; Liedl, T.; Högberg, B.; Graf, F.; Shih, W. M. Selfassembly of DNA into Nanoscale ThreeDimensional Shapes. Nature 2009, 459, 414-418.

5. Dietz, H.; Douglas, S. M.; Shih, W. M. Folding DNA into Twisted and Curved Nanoscale Shapes. Science 2009, 325, 725730 .

6. Han, D.; Pal, S.; Nangreave, J.; Deng, Z.; Liu, Y.; Yan, H. DNA Origami with Complex Curvatures in Three-Dimensional Space. Science 2011, 332, 342-346.

7. Douglas, S. M.; Marblestone, A. H.; Teerapittayanon, S.; Vazquez, A.; Church, G. M.; Shih, W. M. Rapid Prototyping of 3D DNA-Origami Shapes 
with caDNAno. Nucl. Acids Res. 2009, 37, 5001-5006.

8. Jiang, Q.; Song, C.; Nangreave, J.; Liu, X.; Lin, L.; Qiu, D.; Wang, Z.-G.; Zou, G.; Liang, X.; Yan, H.; Ding, B. DNA Origami as a Carrier for Circumvention of Drug Resistance. J. Am. Chem. Soc. 2012, 134, 13396-13403.

9. Douglas, S. M.; Bachelet, I.; Church, G. M. A Logic-Gated Nanorobot for Targeted Transport of Molecular Payloads. Science 2012, 335, 831-834.

10. Halley, P. D.; Lucas, C. R.; McWilliams, E. M.; Webber, M. J.; Patton, R. A.; Kural, C.; Lucas, D. M.; Byrd, J. C.; Castro, C. E. DaunorubicinLoaded DNA Origami Nanostructures Circumvent Drug-Resistant Mechanisms in a Leukemia Model. Small 2016, 12, 308-320.

11. Zhao, Y.-X.; Shaw, A.; Zeng, X.; Benson, E.; Nystrom, A. M.; Hogberg, B. DNA Origami Delivery System for Cancer Therapy with Tunable Release Properties. ACS Nano 2012, 6, 8684-8691.

12. Zhang, Q.; Jiang, Q.; Li, N.; Dai, L.; Liu, Q.; Song, L.; Wang, J.; Li, Y.; Tian, J.; Ding, B.; Du, Y. DNA Origami as an in Vivo Drug Delivery Vehicle for Cancer Therapy. ACS Nano 2014, 8, 6633-6643.

13. Kuzyk, A.; Schreiber, R.; Fan, Z.; Pardatscher, G.; Roller, E.-M.; Högele, A.; Simmel, F. C.; Govorov, A. O.; Liedl, T. DNA-Based Self-Assembly of Chiral Plasmonic Nanostructures with Tailored Optical Response. Nature 2012, 483, 311-314.

14. Kuzyk, A.; Schreiber, R.; Zhang, H.; Govorov, A. O.; Liedl, T.; Liu, N. Reconfigurable 3D Plasmonic Metamolecules. Nat. Mater. 2014, 13, 862-866.

15. Liu, J.; Geng, Y.; Pound, E.; Gyawali, S.; Ashton, J. R.; Hickey, J.; Woolley, A. T.; Harb, J. N. Metallization of Branched DNA
Origami for Nanoelectronic Circuit Fabrication. ACS Nano 2011, 5, 2240-2247.

16. Sun, W.; Boulais, E.; Hakobyan, Y.; Wang, W. L.; Guan, A.; Bathe, M.; Yin, P. Casting Inorganic Structures with DNA Molds. Science 2014, 346, 1258361.

17. Funke, J. J.; Ketterer, P.; Lieleg, C.; Schunter, S.; Korber, P.; Dietz, H. Uncovering the Forces Between Nucleosomes Using DNA Origami. Sci. Adv. 2016, 2, e1600974.

18. Nickels, P. C.; Høiberg, H. C.; Simmel, S. S.; Holzmeister, P.; Tinnefeld, P.; Liedl, T. DNA Origami Seesaws as Comparative Binding Assay. ChemBioChem 2016, 17, 1093-1096.

19. Langecker, M.; Arnaut, V.; Martin, T. G.; List, J.; Renner, S.; Mayer, M.; Dietz, H.; Simmel, F. C. Synthetic Lipid Membrane Channels Formed by Designed DNA Nanostructures. Science 2012, 338, 932-936.

20. Bell, N. A. W.; Engst, C. R.; Ablay, M.; Divitini, G.; Ducati, C.; Liedl, T.; Keyser, U. F. DNA Origami Nanopores. Nano Lett. 2012, 12, 512-517.

21. Göpfrich, K.; Li, C.-Y.; Ricci, M.; Bhamidimarri, S. P.; Yoo, J.; Gyenes, B.; Ohmann, A.; Winterhalter, M.; Aksimentiev, A.; Keyser, U. F. Large-Conductance Transmembrane Porin Made from DNA Origami. ACS Nano 2016, 10, 8207-8214.

22. Hernandez-Ainsa, S.; Bell, N. A.; Thacker, V. V.; Gopfrich, K.; Misiunas, K.; Fuentes-Perez, M. E.; Moreno-Herrero, F.; Keyser, U. F. DNA Origami Nanopores for Controlling DNA Translocation. ACS Nano 2013, 7, 6024-6030.

23. Pfitzner, E.; Wachauf, C.; Kilchherr, F.; Pelz, B.; Shih, W. M.; Rief, M.; Dietz, H. Rigid DNA Beams for High-Resolution Single-Molecule Mechanics. Angew. Chem. Int. Ed. 2013, 52, 7766-7771.

24. Ke, Y.; Meyer, T.; Shih, W. M.; Bellot, G. Regulation at a Distance of Biomolecular 
Interactions Using a DNA Nanoactuator. Nat. Commun. 2016, 7, 10935.

25. Ke, Y.; Lindsay, S.; Chang, Y.; Liu, Y.; Yan, H. Self-Assembled Water-Soluble Nucleic Acid Probe Tiles for Label-Free RNA Hybridization Assays. Science 2008, 319, 180-183.

26. Wang, D.; Fu, Y.; Yan, J.; Zhao, B.; Dai, B.; Chao, J.; Liu, H.; He, D.; Zhang, Y.; Fan, C.; Song, S. Molecular Logic Gates on DNA Origami Nanostructures for MicroRNA Diagnostics. Anal. Chem. 2014, 86, 1932-1936.

27. Martin, T. G.; Bharat, T. A. M.; Joerger, A. C.; Bai, X.-C.; Praetorius, F.; Fersht, A. R.; Dietz, H.; Scheres, S. H. W. Design of a Molecular Support for Cryo-EM Structure Determination. Proc. Natl. Acad. Sci. USA 2016, 113, E7456-E7463.

28. Kauert, D. J.; Kurth, T.; Liedl, T.; Seidel, R. Direct Mechanical Measurements Reveal the Material Properties of ThreeDimensional DNA Origami. Nano Lett. 2011, 11, 5558-5563.

29. Liedl, T.; Högberg, B.; Tytell, J.; Ingber, D. E.; Shih, W. M. Self-Assembly of Three-Dimensional Prestressed Tensegrity Structures from DNA. Nat. Nanotechnol. 2010, 5, 520-524.

30. Gerling, T.; Wagenbauer, K. F.; Neuner, A. M.; Dietz, H. Dynamic DNA Devices and Assemblies Formed by Shape Complementary, Non-Base Pairing 3D Components. Science 2015, 347, 14461452 .

31. Anderson, F. F.; Knudsen, B.; Oliveira, C. L. P.; Frøhlich, R. F.; Krüger, D.; Bungert, J.; Agbandje-McKenna, M.; McKenna, R.; Juul, S.; Veigaard, C.; Koch, J.; Rubinstein, J. L.; Guldbrandtsen, B.; Hede, M. S.; Karlsson, G.; Andersen, A. H.; Pedersen, J. S.; Knudsen, B. R. Assembly and Structural Analysis of a Covalently Closed Nano-scale DNA cage. $\mathrm{Nu}$ cleic Acid Res. 2008, 36, 1113-1119.
32. Zadegan, R. M.; Jepsen, M. D. E.; Thomsen, K. E.; Okholm, A. H.; Schaffert, D. H.; Andersen, E. S.; Birkedal, V.; Kjems, J. Construction of a 4 Zeptoliters Switchable 3D DNA Box Origami. ACS Nano 2012, 6, 10050-10053.

33. Zhou, L.; Marras, A. E.; Castro, C. E. Origami Compliant Nanostructures with Tunable Mechanical Properties. ACS Nano 2014, 8, 27-34.

34. Marras, A. E.; Zhou, L.; Su, H.-J.; Castro, C. E. Programmable Motion of DNA Origami Mechanisms. Proc. Natl. Acad. Sci. USA 2015, 112, 713-718.

35. Marras, A. E.; Zhou, L.; Kolliopoulos, V.; Su, H.-J.; Castro, C. E. Directing Folding Pathways for Multi-Component DNA Origami Nanostructures with Complex Topology. New J. Phys 2016, 18, 055005 .

36. Castro, C. E.; Su, H.-J.; Marras, A. E.; Zhou, L.; Johnson, J. Mechanical Design of DNA Nanostructures. Nanoscale 2015, 7, 5913-5921.

37. Zhou, L.; Marras, A. E.; Su, H.-J.; Castro, C. E. Direct Design of an Energy Landscape with Bistable DNA Origami Mechanisms. Nano Lett. 2015, 15, 1815-1821.

38. Le, J. V.; Luo, Y.; Darcy, M. A.; Lucas, C. R.; Goodwin, M. F.; Poirier, M. G.; Castro, C. E. Probing Nucleosome Stability with a DNA Origami Nanocaliper. ACS Nano 2016, 10, 7073-7084.

39. Funke, J. J.; Ketterer, P.; Lieleg, C.; Korber, P.; Dietz, H. Exploring Nucleosome Unwrapping Using DNA Origami. Nano Lett. 2016, 16, 7891-7898.

40. Mathur, D.; Henderson, E. R. Programmable DNA Nanosystem for Molecular Interrogation. Sci. Rep. 2016, 6, 27413.

41. Šulc, P.; Romano, F.; Ouldridge, T. E.; Rovigatti, L.; Doye, J. P. K.; Louis, A. A. sequence-Dependent Thermodynamics of 
a Coarse-Grained DNA Model. J. Chem. Phys. 2012, 137, 135101.

42. Wagenbauer, K. F.; Wachauf, C. H.; Dietz, H. Quantifying Quality in DNA SelfAssembly. Nat. Commun. 2014, 5, 3691.

43. Bruetzel, L. K.; Gerling, T.; Sedlak, S. M.; Walker, P. U.; Zheng, W.; Dietz, H.; Lipfert, J. Conformational Changes and Flexibility of DNA Devices Observed by Small-Angle X-ray Scattering. Nano Lett. 2016, 16, 4871-4879.

44. Fischer, S.; Hartl, C.; Frank, K.; Rädler, J. O.; Liedl, T.; Nickel, B. Shape and Inter-Helical Spacing of DNA Origami Nanostructures Studied by Small Angle X-ray Scattering. Nano Lett. 2016, 16, 4282-4287.

45. Yoo, J.; Aksimentiev, A. In situ Structure and Dynamics of DNA Origami Determined through Molecular Dynamics Simulations. Proc. Natl. Acad. Sci. USA 2013, 110, 20099-20104.

46. Maffeo, C.; Yoo, J.; Aksimentiev, A. De novo Reconstruction of DNA Origami Structures through Atomistic Molecular Dynamics Simulation. Nucl. Acids Res. 2016, 44, 3013-3019.

47. Castro, C. E.; Kilchherr, F.; Kim, D.-N.; Shiao, E. L.; Wauer, T.; Wortmann, P.; Bathe, M.; Dietz, H. A Primer to Scaffolded DNA Origami. Nat. Methods 2011, 8, 221229.

48. Kim, D.-N.; Kilchherr, F.; Dietz, H.; Bathe, M. Quantitative Prediction of 3D Solution Shape Flexibility of Nucleic Acid Nanostructures. Nucl. Acids Res. 2012, 40, 2862-2868.

49. Pan, K.; Kim, D.-N.; Zhang, F.; Adendorff, M. R.; Yan, H.; Bathe, M. LatticeFree Prediction of Three-Dimensional Structure of Programmed DNA Assemblies. Nat. Commun. 2014, 5, 5578.
50. Sedeh, R. S.; Pan, K.; Adendorff, M. R.; Hallatschek, O.; Bathe, K.-J.; Bathe, M. Computing Nonequilibrium Conformational Dynamics of Structured Nucleic Acid Assemblies. J. Chem. Theory Comput. 2016, 12, 261-273.

51. Morris-Andrews, A.; Rottler, J.; Plotkin, S. S. A Systematically CoarseGrained Model for DNA and Its Predictions for Persistence Length, Stacking, Twist, and Chirality. J. Chem. Phys. 2010, 132, 035105 .

52. Zhou, L.; Marras, A. E.; Castro, C. E.; $\mathrm{Su}$, H.-J. Pseudorigid-Body Models of Compliant DNA Origami Mechanisms. J. Mechanisms Robotics 2016, 8, 051013.

53. Savelyev, A.; Papoian, G. A. Chemically Accurate Coarse-Graining of DoubleStranded DNA. Proc. Natl. Acad. Sci. USA 2010, 10\%, 20340-20345.

54. Dans, P. D.; Zeida, A.; Machado, M. R.; Pantano, S. A Coarse-Grained Model for Atomic-Detailed DNA Simulations with Explicit Electrostatics. J. Chem. Theory Comput. 2010, 6, 1711-1725.

55. Hinckley, D. M.; Freeman, G. S.; Whitmer, J. K.; de Pablo, J. J. An Experimentally-Informed Coarse-Grained 3-Site-per-Nucleotide Model of DNA: Structure, Thermodynamics, and Dynamics of Hybridization. J. Chem. Phys. 2013, 139, 144903.

56. Korolev, N.; Luo, D.; Lyubartsev, A. P.; Nordensklöld, L. A Coarse-Grained DNA Model Parameterized from Atomistic Simulations by Inverse Monte Carlo. Polymers 2014, 6, 1655-1675.

57. Markegard, C. B.; Fu, I. W.; Reddy, K.; Nguyen, H. D. Coarse-Grained Simulation Study of Sequence Effects on DNA Hybridization in a Concentrated Environment. J. Phys. Chem. B 2015, 119, 18231834. 
58. Ouldridge, T. E.; Louis, A. A.; Doye, J. P. K. Structural, Mechanical and Thermodynamic Properties of a Coarse-Grained DNA Model. J. Chem. Phys. 2011, 134, 085101.

59. Snodin, B. E. K.; Randisi, F.; Mosayebi, M.; Šulc, P.; Schreck, J. S.; Romano, F.; Ouldridge, T. E.; Tsukanov, R.; Nir, E.; Louis, A. A.; Doye, J. P. K. Introducing Improved Structural Properties and Salt Dependence into a Coarse-Grained Model of DNA. J. Chem. Phys. 2015, 142, 234901.

60. Ouldridge, T. E.; Louis, A. A.; Doye, J. P. K. DNA Nanotweezers Studied with a Coarse-Grained Model of DNA. Phys. Rev. Lett 2010, 104, 178101.

61. Doye, J. P. K.; Ouldridge, T. E.; Louis, A. A.; Romano, F.; Šulc, P.; Matek, C.; Snodin, B. E. K.; Rovigatti, L.; Schreck, J. S.; Harrison, R. M.; Smith, W. P. J. Coarse-Graining DNA for Simulations of DNA Nanotechnology. Phys. Chem. Chem. Phys. 2013, 15, 2039520414.

62. Ouldridge, T. E.; Hoare, R. L.; Louis, A. A.; Doye, J. P. K.; Bath, J.; Turberfield, A. J. Optimizing DNA nanotechnology through Coarse-Grained Modelling: A Two-Footed DNA Walker. ACS Nano 2013, 7, 2479-2490.

63. Šulc, P.; Ouldridge, T. E.; Romano, F.; Doye, J. P. K.; Louis, A. A. Simulating a Burnt-Bridges DNA Motor with a CoarseGrained DNA Model. Natural Computing 2014, 13, 535-547.

64. Kočar, V.; Schreck, J.; Čeru, S.; Gradišar, H.; Bašić, N.; Pisanki, T.; Doye, J. P. K.; Jerala, R. Design Principles for Rapid Folding of Knotted DNA Nanostructures. Nat. Commun 2016, \%, 10803

65. Snodin, B. E. K.; Romano, F.; Rovigatti, L.; Ouldridge, T. E.; Louis, A. A.;
Doye, J. P. K. Direct Simulation of the SelfAssembly of a Small DNA Origami. ACS Nano 2016, 10, 1724-1737.

66. Schreck, J. S.; Romano, F.; Zimmer, M. H.; Louis, A. A.; Doye, J. P. K. Characterizing DNA star-tile-based nanostructures using a coarse-grained model. ACS Nano 2016, 10, 4236-4247.

67. Shi, Z.; Castro, C. E.; Arya, G. Conformational Dynamics of MechanicallyCompliant DNA Nanostructures from Coarse-Grained Molecular Dynamics Simulations. ACS Nano 2017, 11, 4617-4630.

68. Romano, F.; Chakraborty, D.; Doye, J. P. K.; Ouldridge, T. E.; Louis, A. A. Coarse-Grained Simulations of DNA Overstretching. J. Chem. Phys. 2013, 138, 085101.

69. Matek, C.; Ouldridge, T. E.; Doye, J. P. K.; Louis, A. A. Plectoneme Tip Bubbles: Coupled Denaturation and Writhing in Supercoiled DNA. Sci. Rep. 2015, 5, 7655.

70. Snodin, B. E. K. Simulating Large DNA Nanostructures with a Coarse-Grained Model. Ph.D. thesis, University of Oxford, 2015 .

71. Schreck, J. S.; Ouldridge, T. E.; Romano, F.; Louis, A. A.; Doye, J. P. K. Characterizing the Bending and Flexibility Induced by Bulges in DNA Duplexes. J. Chem. Phys. 2015, 142, 165101.

72. Marras, A. E.; Zhou, L.; Su, H.-J.; Castro, C. E. Programmable Motion of DNA Origami Mechanisms. Proc. Natl. Acad. Sci. USA 2015, 112, 713-718.

73. Ruf, A.; Horaud, R. Visual Servoing of Robot Manipulators Part I: Projective Kinematics. The International Journal of Robotics Research 1999, 18, 1101-1118.

74. Zhou, L.; Marras, A. E.; Castro, C. E.; $\mathrm{Su}$, H.-J. Projection Kinematic Analysis of DNA Origami Mechanisms Based on 
a Two-Dimensional TEM Image. Mechanisms and Machine Theory 2017, 109, 2238.

75. Rovigatti, L.; Šulc, P.; Reguly, I. Z.; Romano, F. A Comparison between Parallelization Approaches in Molecular Dynamics Simulations on GPUs. J. Comput. Chem. 2015, 36, 1-8.

76. Russo, J.; Tartaglia, P.; Sciortino, F. Reversible Gels of Patchy Particles: Role of the Valence. J. Chem. Phys. 2009, 131, 014504 .

\section{TOC Entry}




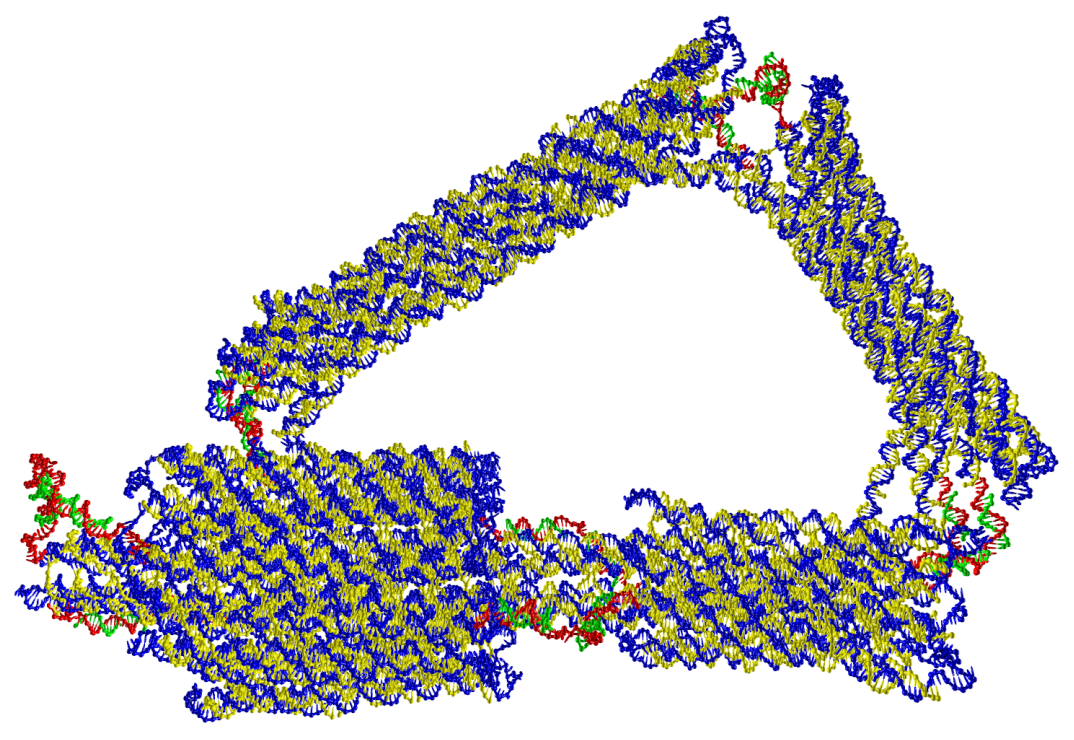

